\title{
Near-infrared spectroscopy as a complementary age grading and species identification tool for African malaria vectors
}

\author{
Maggy Sikulu*1,2, Gerry F Killeen2,3, Leon E Hugo', Peter A Ryan', Kayla M Dowell6, Robert A Wirtz, Sarah J Moore2,5 \\ and Floyd E Dowell6
}

\begin{abstract}
Near-infrared spectroscopy (NIRS) was recently applied to age-grade and differentiate laboratory reared Anopheles gambiae sensu strico and Anopheles arabiensis sibling species of Anopheles gambiae sensu lato complex. In this study, we report further on the accuracy of this tool for simultaneously estimating the age class and differentiating the morphologically indistinguishable An. gambiae s.s. and An. arabiensis from semi-field releases and wild populations. Nine different ages $(1,3,5,7,9,11,12,14,16 \mathrm{~d})$ of An. arabiensis and eight different ages $(1,3,5,7,9,10,11,12 \mathrm{~d})$ of $\mathrm{An}$. gambiae s.s. maintained in $250 \times 60 \times 40 \mathrm{~cm}$ cages within a semi-field large-cage system and 105 wild-caught female An. gambiae s.l., were included in this study. NIRS classified female An. arabiensis and An. gambiae s.s. maintained in semi-field cages as $<7$ d old or $\geq 7$ d old with $89 \%(n=377)$ and $78 \%(n=327)$ accuracy, respectively, and differentiated them with $89 \%(n=704)$ accuracy. Wild caught An. gambiae s.l. were identified with $90 \%$ accuracy $(n=105)$ whereas their predicted ages were consistent with the expected mean chronological ages of the physiological age categories determined by dissections. These findings have importance for monitoring control programmes where reduction in the proportion of older mosquitoes that have the ability to transmit malaria is an important outcome.
\end{abstract}

\section{Findings}

Vector survival is recognised as one of the most imperative determinants of vector-borne pathogen transmission. For example, malaria vectors can only transmit malaria parasites when they are at least 10 days old because of the lengthy period required for Plasmodium parasite development in the mosquito [1]. Traditionally, scientists relied upon observations of morphological changes in the reproductive system of female mosquitoes to estimate their physiological age [2-6] and to assess disease transmission potential $[7,8]$. However, these well-established age measurement techniques are labour intensive and they engage highly skilled personnel. These disadvantages render the techniques unsuitable for assessing age distribution at an operational level in large scale, community-randomized trials. New tools are therefore required that can effectively, rapidly, and accurately assess the ages

* Correspondence: maggysikulu@yahoo.com

${ }^{1}$ Griffith Medical Research College, a joint program of Griffith University and the Queensland Institute of Medical Research, Herston, QLD, 4006, Australia Full list of author information is available at the end of the article of large numbers of mosquitoes as existing priority intervention technologies are scaled up $[9,10]$ and new complementary approaches are developed and evaluated.

Furthermore, while accurate polymerase chain reaction methods do exist for differentiating sibling species such as those from the An. gambiae and Anopheles funestus complex in Africa [11-13], these methods are also somewhat laborious and expensive, limiting the numbers of mosquitoes which can be rigorously classified in most field studies. A convenient high throughput technique for simultaneously classifying and estimating the age of large numbers of mosquitoes would therefore enable biodemographic surveys of vector populations, and the impact of specific interventions upon them, on unprecedented scales.

Near-infrared spectroscopy (NIRS) uses the near-infrared region of the electromagnetic spectrum to quantitatively measure organic compounds e.g. O-H, N-H and C$\mathrm{O}$ functional groups in biological samples. The spectrum collected is a result of the near-infra-red energy absorbed by a sample and is proportional to the amount of these 
functional groups present in samples. It is expected that a unique spectrum would be obtained for different age classes as well as different species of mosquitoes since it has been demonstrated that cuticular hydrocarbons change with age of mosquitoes [14] and that An. arabiensis have more water content in their body than An. gambiae s.s. [15]. After calibrations have been developed, the technique is very simple, requiring very little training or expertise. Whole insects are placed below a fibre-optic probe, a spectrum collected, and the age and species predicted from stored calibrations. Advantages of this technique are that insects can be scanned non-destructively, no sample preparation is required, and results are obtained in a few seconds.

NIRS was recently applied successfully to age and distinguish laboratory reared An. gambiae s.s. from An. arabiensis [16]. However, this study showed that additional data were needed to further develop calibrations and that additional field validation was needed. Herein we report on evaluation of the accuracy of this NIRS technique to estimate the ages and classify semi-field-maintained members of the An. gambiae s.l. complex, namely $A n$. gambiae s.s. and An. arabiensis. We also further validated the accuracy of NIRS for differentiating wild caught $A n$. gambiae s.s. from An. arabiensis and made a preliminary assessment of whether it might be useful for age grading wild-caught An. gambiae s.l.

An. gambiae s.s. (colony established in 1996 from Njage village, Kilombero, Tanzania) and An. arabiensis (colony established in 2007 from Sagamaganga village, Kilombero, Tanzania) were reared in the semi-field system established in the Ifakara Health Institute [17]. To avoid variance in adult emergence rates and development characteristics arising from environmental differences, larvae and pupae of $A n$. gambiae s.s. and An. arabiensis were reared under their usual rearing conditions in their respective laboratory and semi-field colonies. Pupae of both siblings were transferred into small cages measuring $40 \times 40 \times 30 \mathrm{~cm}$ for emergence. Adult mosquitoes were moved to larger netted cages measuring $250 \times 60 \times 40 \mathrm{~cm}$ at day 0 (within a day of emergence). Adult cages for both sibling species were maintained within the semi-field system. A total of four cages for each species, representing four different age cohorts were reared. In addition to paper cups lined with moist filter papers as oviposition sites, two clay pots lined with a black cloth were positioned inside each cage to provide cool resting sites for these mosquitoes. Adult females were blood-fed twice a week by inserting a human (volunteer) arm inside the cage each time for 15 minutes (Ethical clearance No. IHRDC/EC4/CL.N96/2004) and provided with fresh 10\% glucose solution daily. Wild mosquitoes were collected from Njage village, Kilombero, Tanzania using CDCLight traps over two consecutive nights.
Prior to scanning, mosquitoes were anesthetised using chloroform. At least 40 females of each sibling species in each age cohort were scanned using an ASD Lab Spec 5000 (Boulder, Colorado) NIR spectrometer. Nine ages (1, 3, 5, 7, 9, 11, 12, 14, 16 d) of An. arabiensis and eight ages $(1,3,5,7,9,10,11,12 \mathrm{~d})$ of An. gambiae s.s. from the semi field system were scanned. 105 wild caught An. gambiae s.l. were scanned for age and the sibling species identified as soon as they were collected from the field.

The scanning protocol has previously been described elsewhere [16]. Soon after the NIRS scans, $27 \%(n=28)$ of the wild An. gambiae s.l. were dissected to determine their parity and ovarian development status [5]. Polymerase Chain Reaction was used to validate the accuracy of NIRS for differentiating wild An. arabiensis from $A n$. gambiae s.s. [18].

A calibration model developed from partial least squares regression cross-validation [16] was used to predict the age and differentiate semi-field reared and wild caught An. gambiae s.l. sibling species. This was achieved by using the semi-field reared data in the calibration models. The improved species identification model included the following ages: 1, 5, 7, 11, $12 \mathrm{~d}$ for $A n$. arabiensis and. 1, 3, 5, 9, $10 \mathrm{~d}$ for An. gambiae s.s. For differentiating species, An. arabiensis was assigned a value of "1", and $A n$. gambiae s.s. assigned a value of "2". Samples were then classed depending on whether they were predicted above or below 1.5 classification cut off point.

All mosquitoes that were identified as $<7$ or $\geq 7$ days old by NIRS were classified as young or old, respectively. Out of the 704 An. gambiae s.s. and An. arabiensis reared under the semi-field conditions, $84 \%$ of them were accurately predicted as young or old (Figure 1A and 1B). Also, $89 \%(\mathrm{n}=377)$ of the An. arabiensis (Figure 1A) and $78 \%$ ( $\mathrm{n}=327$ ) of the An. gambiae s.s. (Figure 1B) were accurately ranked as young or old.

The cross-validation technique distinguished the two sibling species reared in the semi-field system with $90 \%$ accuracy (Figure 1C) and the rest of the species categories that were not included in the initial calibration model (An. arabiensis 3, 9, $14 \mathrm{~d}$ and An. gambiae s.s. 7, 11, $12 \mathrm{~d}$ ) were classified with $88 \%$ accuracy.

For the wild specimens, NIRS predictions illustrated that $92 \%$ of the 105 Anopheles collected over the two sampling nights were An. gambiae s.s. (Figure 2A). To validate the results, 103 female An. gambiae s.l. were analyzed by PCR. An amplification success rate of $83 \%$ was obtained. PCR confirmed that NIRS had predicted the two sibling species with $90 \%$ accuracy. All the specimens predicted by NIRS to be An. gambiae s.s. were confirmed by PCR as correctly identified with the exception of those that could not be determined by PCR. Moreover, all the successful amplifications obtained were determined as An. gambiae s.s. while 2 of the PCR undetermined sam- 

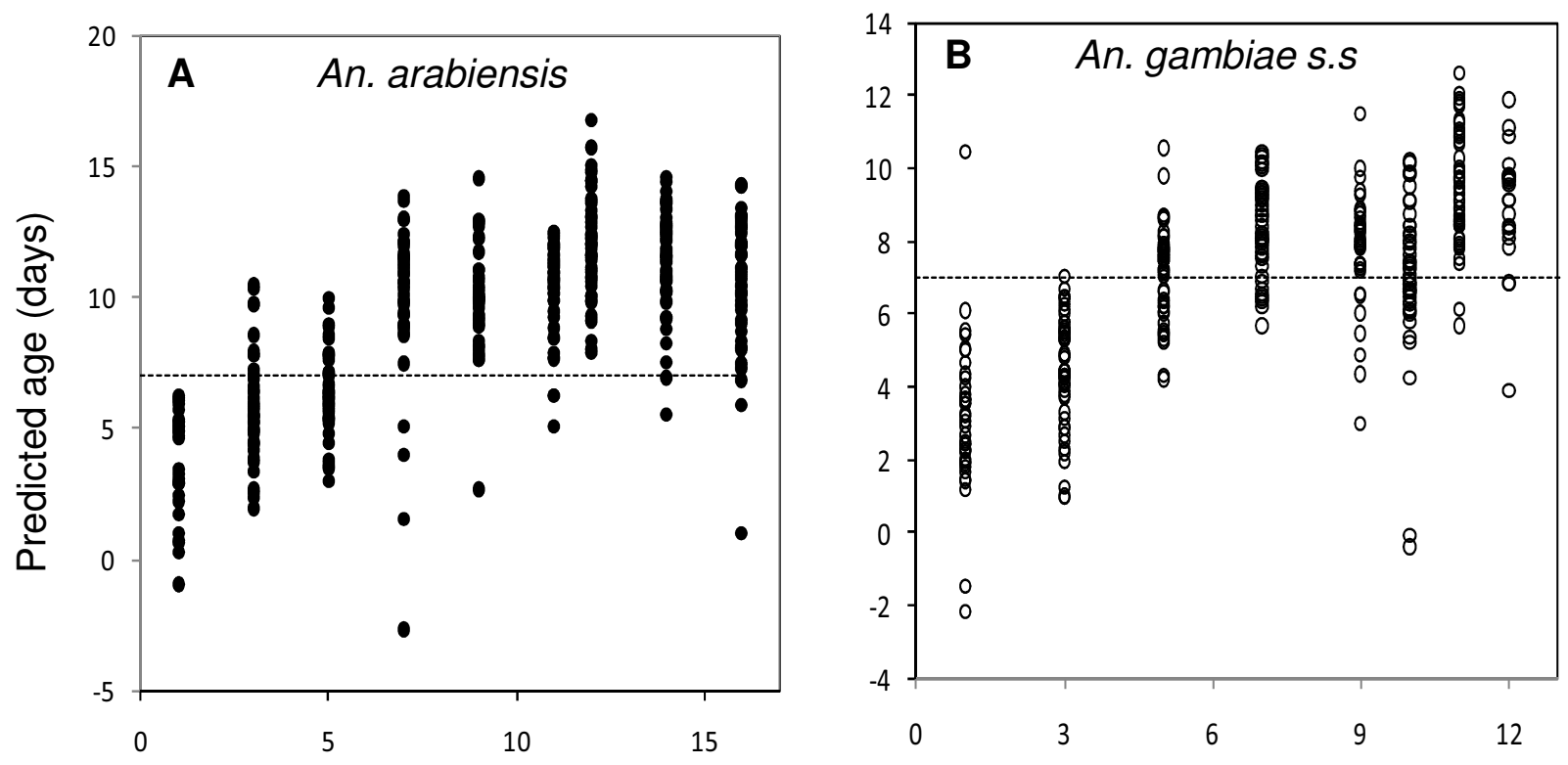

Actual age (days)

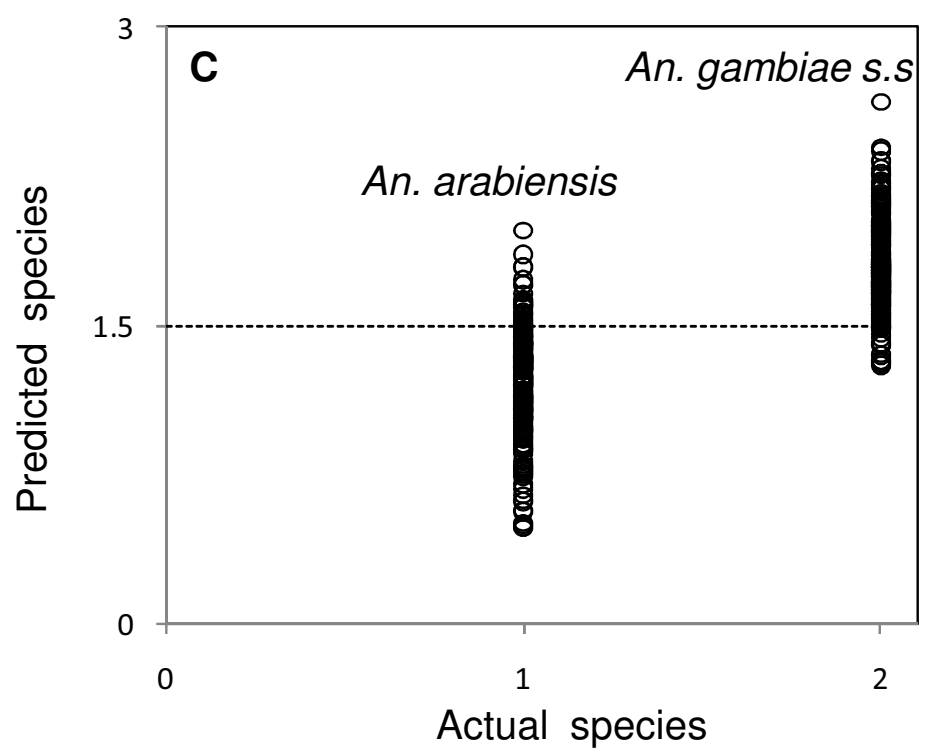

Figure 1 NIRS age and species prediction for semi-field reared An. arabiensis and An. gambiae s.s. as determined from a cross-validation Panel A (An. arabiensis) and B (An. gambiae s.s.) indicate the actual age against the predicted age. The horizontal dotted line in Panel A and B separates young mosquitoes ( $<7 \mathrm{~d}$ ) from old ones $(\geq 7 \mathrm{~d})$. Panel $\mathrm{C}$ shows the actual species $(1=A n$. arabiensis and $2=A n$. gambiae s.s.) against the predicted species for 1, 5, 7, 11, 12 days old An. arabiensis and 1, 3, 5, 9, 10 days old An. gambiae s.s. The dotted line in panel $\mathrm{C}$ is the classification cut off point for the two sibling species (<1.5 for An. arabiensis and $>1.5$ for An. gambiae s.s.).

ples had been identified as An. arabiensis by NIRS. However, those predicted as An. arabiensis (10\%) by NIRS but determined as An. gambiae s.s. by PCR, were very close to the 1.5 classification cut off point of the two sibling spe- cies. The confidence level associated with the NIRS classifications could be further increased by excluding any samples predicted as close to 1.5. The user would then have greater confidence that the remaining samples were 
correctly classified. A summary of the accuracy of age and species predictions for semi-field and wild An. arabiensis and An. gambiae s.s. by the NIRS is provided in table 1.

NIRS age predictions for wild caught An. gambiae s.l. revealed a comparatively similar age structure for mosquitoes collected in two consecutive nights from the same houses (Figure 2B). Less than $22 \%$ of the mosquitoes collected each night were estimated as $\geq 7$ days old. Figure 3 illustrates NIRS predicted ages of wild caught mosquitoes for which their ovary dissections were classified as underdeveloped (Christophers' stages $\leq \mathrm{IIm}$ ) nulliparous, fully developed (Christophers' stages >IIm) nulliparous or parous for the first night $(n=13)$ and second night $(\mathrm{n}=15)$ [5]. These results are consistent with mean chronological ages of nulliparous mosquitoes as determined previously by dissections $[4,5]$. However, the relatively low mean predicted age of parous mosquitoes relative to historical reports [4], is consistent with reduced adult female survivorship due to high insecticidal net coverage in this area (Russell et al., unpublished). These results also suggest that the population age structure from which these mosquitoes were collected from was quite stable for both nights.

Studies involving vector age assessments are crucial for determining the success or failure of any malaria control strategy that targets mosquito life span [19-22]. This study represents an extension of previous work [16] on laboratory and field specimens which indicated that additional data were needed to further develop calibrations and that further field validation was essential to verify the results. We have established and confirmed that NIRS can be applied to rapidly distinguish young from old $A n$. gambiae s.s. and An. arabiensis and separate these morphologically similar species in areas where they do not occur sympatrically with other sibling species. Although
NIRS was applied to age grade a relatively small sample of wild-caught specimens, insights were gained into the stability of the mosquito population age structure. Largescale studies could potentially enable far more ambitious studies of malaria vector ecology, as well as the impact of control interventions upon vector bio-demography and transmission potential.

NIRS is non-destructive, rapid, and is associated with minimal sample processing costs after an initial outlay (approximately $\$ 40,000$ ) for a NIR spectrometer. On the basis that NIRS can rapidly handle a large set of data with minimal labour and resources, the overall cost of using it on a large scale is dramatically reduced in the long run. Comparatively, the cost of cuticular hydrocarbon analysis depends largely on the accessibility of GC/FID or GC/MS instrumentation and, if the analysis is outsourced, can reach over US\$50 per sample while the cost of transcriptional mosquito age grading has been estimated to be between \$US7.5 to 10 per sample [23,24]. Additionally, current age assessment tools are not conducive to the rapid assessment of mosquito population age structure on a large scale while standard PCR [11] and multiplex PCR $[12,13]$ for differentiating morphologically indistinguishable species is costly and time consuming. In terms of speed, NIRS was more than 10 times faster than parity dissections to determine physiological age. It required less than 15 seconds to scan one mosquito using NIRS and the spectrum obtained was analysed for both age and sibling species identification. Additionally, only one out of four technicians involved in this study could perform dissections to determine parity and these required approximately 3-5 minutes per mosquito. PCR reactions to determine sibling species required 2 people who took on average 5 hours working on 50 samples. Comparatively, it took less than 30 minutes to train 2 people to operate NIRS.

Table 1: The accuracy of NIRS for predicting the age and species of semi-field and wild An. arabiensis and An. gambiae s.s.

\begin{tabular}{|c|c|c|c|c|c|c|c|c|}
\hline \multirow[t]{3}{*}{ Condition } & \multicolumn{4}{|l|}{ An. arabiensis } & \multicolumn{4}{|c|}{ An. gambiae s.s. } \\
\hline & \multicolumn{2}{|l|}{ Age } & \multicolumn{2}{|l|}{ Species ID } & \multicolumn{2}{|l|}{ Age } & \multicolumn{2}{|l|}{ Species ID } \\
\hline & No. scanned & \%correct & No. scanned & \%correct & No. scanned & \%correct & No. scanned & \%correct \\
\hline Semi field & 377 & 89 & 202 & $89^{*}$ & 327 & 78 & 201 & $91^{*}$ \\
\hline Wild & 11 & $N / A^{* *}$ & 11 & $N / A^{* * *}$ & 94 & $N A^{* *}$ & 94 & 90 \\
\hline $\begin{array}{l}\text { ID-Identific } \\
\text { No. -Numbe } \\
{ }^{*} \text { Accuracy } \\
{ }^{* *} \text { Accuracy } \\
{ }^{* * *} \text { Accola }\end{array}$ & $\begin{array}{l}5,7,11 \text { and } 12 \\
d \text { only be verifi }\end{array}$ & $\begin{array}{l}\mathrm{d} \text { An. arabie } \\
\text { ed on a prop }\end{array}$ & $\begin{array}{l}\text { nd } 1,3,5,9 \text { an } \\
\text { n of the samp }\end{array}$ & $\begin{array}{l}10 \mathrm{~d} \text { An. ga } \\
\text { es by parity }\end{array}$ & $\begin{array}{l}\text { e s.s. ages incl } \\
\text { ctions (see fig }\end{array}$ & $\begin{array}{l}\text { ded in the pr } \\
\text { e 3) }\end{array}$ & usly developed & nodel. \\
\hline
\end{tabular}



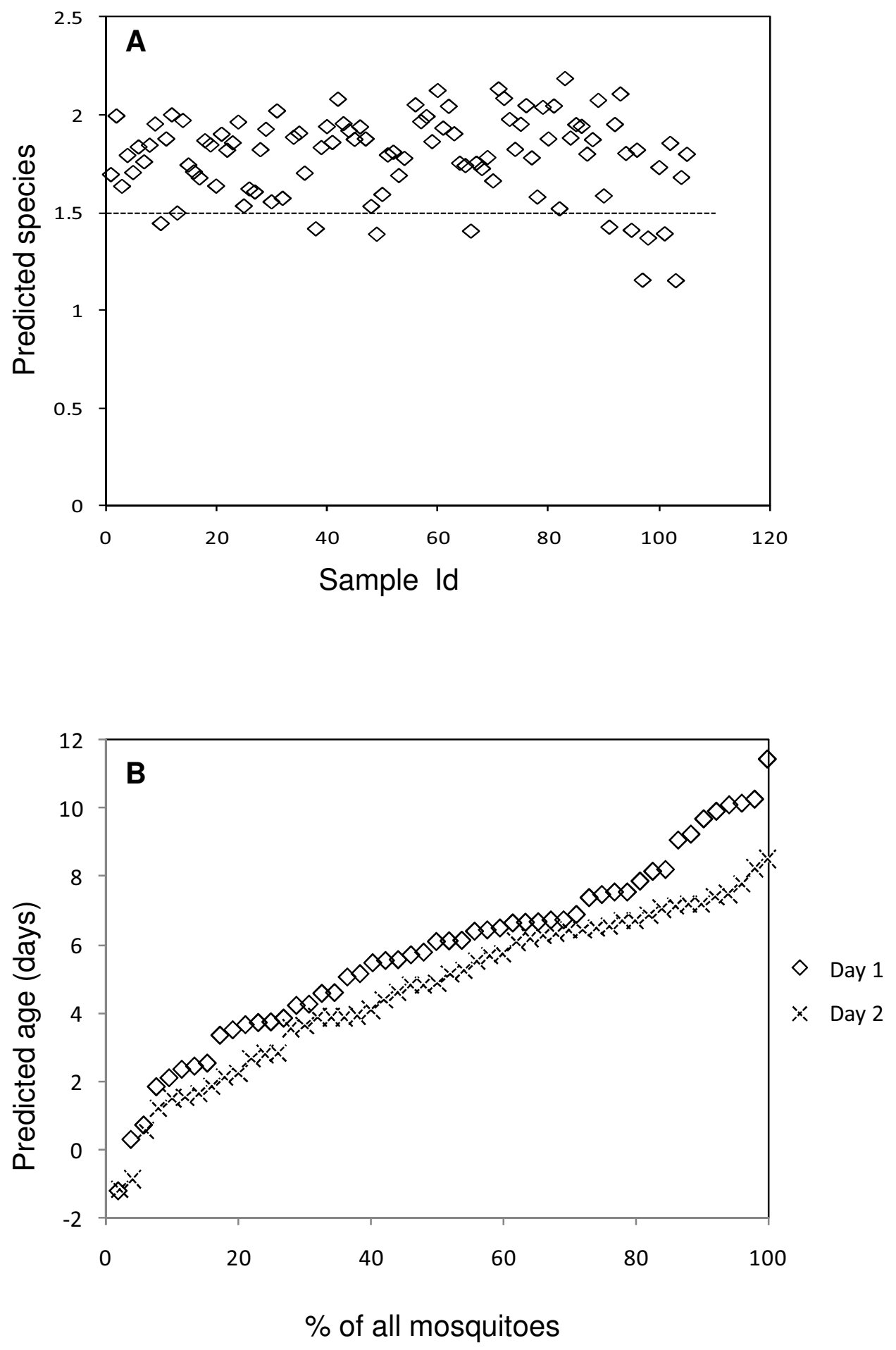

Figure 2 NIRS age and species predictions for wild-caught mosquitoes. Panel A indicates that $92 \%$ of all the wild mosquitoes were An. gambiae S.S. as predicted by NIRS. The dotted line in panel A is the classification cut off point for An. gambiae s.s. and An. arabiensis as predicted by NIRS $(<1.5$ for An. arabiensis and >1.5 for An. gambiae s.s.). Panel B shows the predicted cumulative age structure of wild mosquitoes from Njage collected in two consecutive nights. 


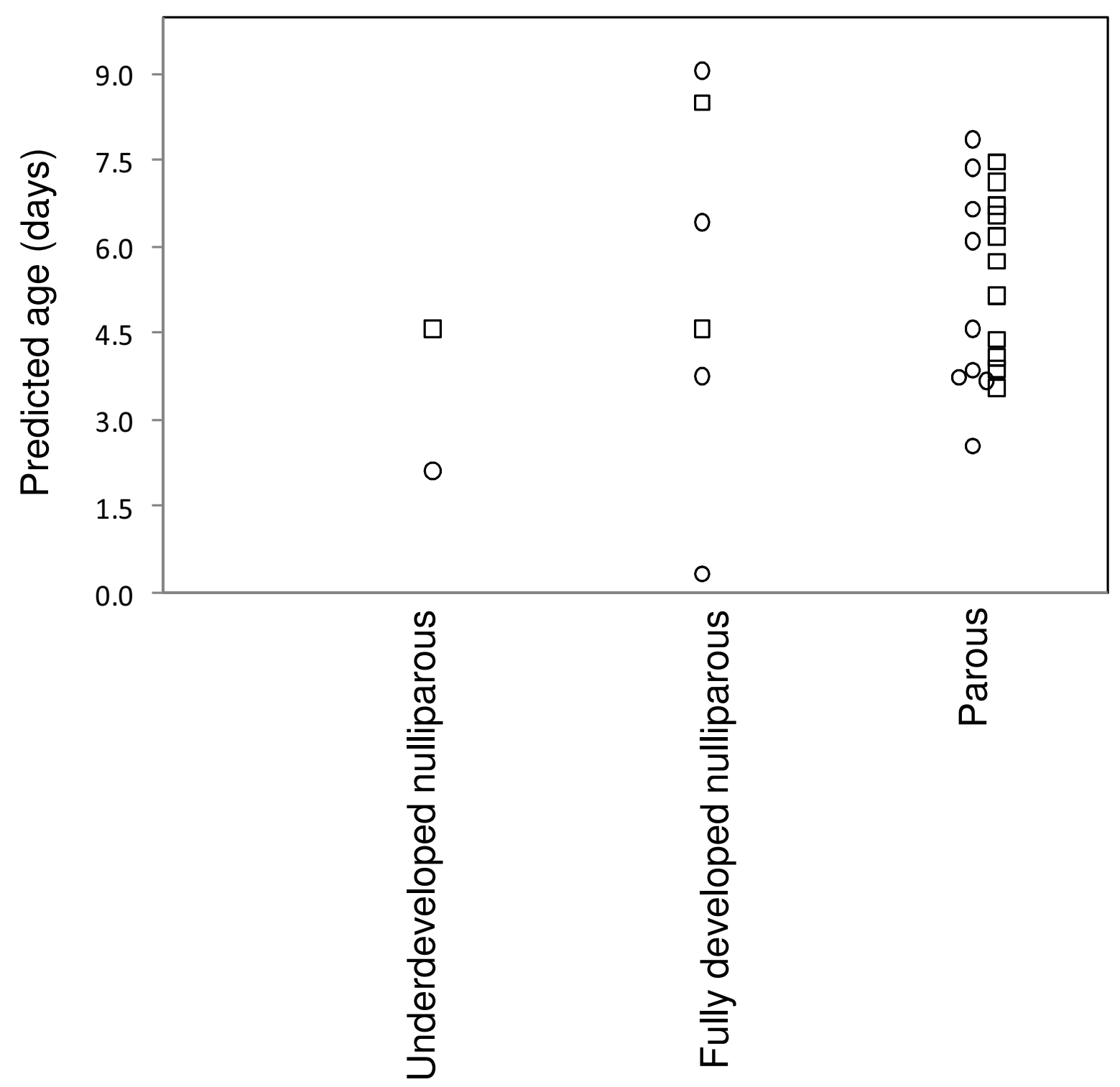

Figure 3 NIRS age prediction of wild mosquitoes that were classified by parity dissections as underdeveloped (Christophers' stages $\leq$ IIm) nulliparous, fully developed (Christophers' stages $>\mathrm{IIm}$ ) nulliparous and parous for the first night (represented by circles) and the second night (represented by squares) [5].

Although this study provides grounds for optimism, further work is clearly needed. Refinements to the method, such as the capacity to perform NIRS on preserved samples will practically facilitate large studies particularly in areas where field specimens must be preserved for future analysis. While there is a need for further age grading methods to determine population age structure on a finer scale, the value of NIRS is in the unique capacity to rapidly identify changes to mosquito population demography. Further studies are required to determine the capacity of this tool to differentiate and age grade other morphologically indistinguishable species in the An. gambiae complex and mosquitoes in other genera.

\section{Competing interests}

All authors declare that they have no competing interests.

\section{Authors' contributions}

MS drafted the manuscript; SJM, GFK, RAW, FED conceived the study; MS, LEH, PAR, SJM, FED and GFK designed the experiments; MS, FED, and KMD ran the experiments; FED analysed data; FED, GFK, SJM, LEH, PAR and RAW reviewed the manuscript. All authors read and approved the final manuscript. 


\section{Acknowledgements}

We thank the insectary staff at IHI; Paulina Kasanga, and Ally Daraja for rearing mosquitoes and Hassan Mtambala, Peter Pazia, Daniel Lugiko, Nuru Nchimbi and Japheth Kihonda for their technical assistance. We thank residents of Njage village for allowing us to trap mosquitoes in their houses. We acknowledge the Griffith Medical Research College and the Science, Engineering, Environment and Technology group, Griffith University, for providing a PhD scholarship to MS. This study was supported by the Bill \& Melinda Gates Foundation (awards 51431 and 45114) as well as a Research Career Development Fellowship (076806) provided to GFK by the Wellcome Trust and IAEA fellowship funding provided to FED.

\section{Author Details}

'Griffith Medical Research College, a joint program of Griffith University and the Queensland Institute of Medical Research, Herston, QLD, 4006, Australia, Iffakara Health Institute, Biomedical and Environmental Thematic Group, Ifakara and Dar es Salaam Branches, United Republic of Tanzania, ${ }^{2}$ Liverpool School of Tropical Medicine and Hygiene, Vector Group, Liverpool, UK, ${ }^{4}$ Centers for Disease Control and Prevention, Atlanta, Georgia, USA, ${ }^{5}$ Disease Control and Vector Biology Unit, London School of Hygiene and Tropical Medicine, London, UK and 6 USDA ARS, Engineering and Wind Erosion Research Unit, Center for Grain and Animal Health Research, Manhattan, Kansas, USA

Received: 29 March 2010 Accepted: 4 June 2010 Published: 4 June 2010

\section{References}

1. Beier CJ: Malaria parasite development in mosquitoes. Annu Rev Entomol 1998, 43:519-543.

2. Detinova T: Age-grouping methods in Diptera of medical importance, with special reference to some vectors of malaria. Monogr Ser World Health Organ 1962, 47:13-191.

3. Polovodova VP: Age changes in ovaries of Anopheles and methods of determination of age composition in mosquito population. Med Parazit (Mosk) 1941, 10:387.

4. Gillies M, Wilkes T: A study of the age composition of Anopheles gambiae Giles and A. funestus Giles in north-eastern Tanzania. Bull Entomol Res 1965, 56:237-262.

5. Gillies M: A modified technique for the age-grading of populations of Anopheles gambiae. Ann Trop Med Parasit 1958, 52:261-273.

6. Gillies M: Studies on the dispersion and survival of Anopheles gambiae in East Africa, by means of marking and release experiments. Bull Entomol Res 1961, 52:99-127.

7. Garret-Jones C: Prognosis for interuption of malaria transmission through assessment of the mosquito's vectorial capacity. Nature 1964, 204:1173-1175.

8. Dye C: The analysis of parasite transmission by bloodsucking Insects. Annu Rev Entomol 1992, 37:1-19.

9. Sharp BL, Kleinschmidt I, Streat E, Maharaj R, Barnes KI, Durrheim DN, Ridl FC, Morris N, Seocharan I, Kunene S, La grange JJP, Mthembu JD, Maartens F, Martin CL, Barreto A: Seven years of regional malaria control collaboration - Mozambique, South Africa, and Swaziland. Am J Trop Med Hyg 2007, 76:42-47.

10. Noor AM, Mutheu JJ, Tatem AJ, Hay SI, Snow RW: Insecticide-treated net coverage in Africa: mapping progress in 2000-07. The Lancet 2009, 373:58-67.

11. Scott J, Brogdon W, Collins F: Identification of single specimens of the Anopheles gambiae complex by the polymerase chain reaction. Am J Trop Med Hyg 1993, 49:520-529.

12. Vezenegho S, Bass C, Puinean M, Williamson M, Field L, Coetzee M, Koekemoer L: Development of multiplex real-time PCR assays for identification of members of the Anopheles funestus species group. Malar J 2009, 8:282

13. Bass C, Williamson M, Wilding C, Donnelly M, Field L: Identification of the main malaria vectors in the Anopheles gambiae species complex using a TaqMan real-time PCR assay. Malar J 2007, 6:155.

14. Caputo B, Dani RF, Horne LG, Petrarca V, Turillazzi S, Coluzzi M, Priestman AA, Torre A: Identification and composition of cuticular hydrocarbons of the major Afrotropical malaria vector Anopheles gambiae s.s.
(Diptera: Culicidae): analysis of sexual dimorphism and age-related changes. J Mass Spectrom 2005, 40:1595-1604.

15. Gray EM, Bradley T: Physiology of dessication resistance in Anopheles gambiae and Anopheles arabiensis. Am J Trop Med Hyg 2005, 73:553-559.

16. Mayagaya VS, Michel K, Benedict MQ, Killeen GF, Wirtz RA, Ferguson HM, Dowell FE: Non-destructive determination of age and species of Anopheles gambiae s.l. using Near-infrared spectroscopy. Am J Trop Med Hyg 2009, 81:622-630

17. Ferguson H, Ng'habi K, Walder T, Kadungula D, Moore S, Lyimo I, Russell T, Urassa H, Mshinda H, Killeen G, Knols B: Establishment of a large semifield system for experimental study of African malaria vector ecology and control in Tanzania. Malar J 2008, 7:158

18. Scott JA, Brogdon WG, Collins FH: Identification of single specimens of the Anopheles gambiae complex by the polymerase chain reaction. Am J Trop Med Hyg 1993, 49:520-529.

19. Scholte EJ, Knols BGJ, Samson RA, Takken W: Entomopathogenic fungi for mosquito control: A review. J Insect Sci 2004, 4:19.

20. Scholte EJ, Knols BGJ, Takken W: Infection of the malaria mosquito Anopheles gambiae with the entomopathogenic fungus Metarhizium anisopliae reduces blood feeding and fecundity. I Invertebr Pathol 2005, 91:43-49.

21. Blanford S, Chan BHK, Jenkins N, Sim D, Turner RJ, Read AF, Thomas MB: Fungal pathogen reduces potential for malaria transmission. Science 2005, 308:1638-1641.

22. Brownstein JS, Hett E, O'Neill SL: The potential of virulent Wolbachia to modulate disease transmission by insects. J Invertebr Pathol 2003, 84:24-29.

23. Hugo EL, Peter EC, Petrina HJ, Luke PR, Brian HK, Peter AR, Scott AR, Scott LON: Field Validation of a Transcriptional Assay for the Prediction of Age of Uncaged Aedes aegypti Mosquitoes in Northern Australia. PLOS Negl Trop Dis 2010, 4:e608.

24. Cook PE, Hugo LE, Iturbe-Ormaetxe I, Williams CR, Chenoweth SF, Ritchie SA, Ryan PA, Kay BH, Blows MW, O'Neill SL: Predicting the age of mosquitoes using transcriptional profiles. Nat Protoc 2007, 2:2796-2806.

doi: 10.1186/1756-3305-3-49

Cite this article as: Sikulu et al., Near-infrared spectroscopy as a complementary age grading and species identification tool for African malaria vectors Parasites \& Vectors 2010, 3:49

\section{Submit your next manuscript to BioMed Central and take full advantage of:}

- Convenient online submission

- Thorough peer review

- No space constraints or color figure charges

- Immediate publication on acceptance

- Inclusion in PubMed, CAS, Scopus and Google Scholar

- Research which is freely available for redistribution 